\title{
Risks Assessment of Nurses based on Aggressive Behaviors of Patients in Department of Psychiatry and the Research on Nursing Intervention Effects
}

\author{
Guolian Zhan * \\ Mental Nursing Center of Xiaoshan Hospital of Zhejiang, Hangzhou, Zhejiang 311200, China
}

\begin{abstract}
Objective: To assess the risks of nurses based on aggressive behaviors of patients in Department of Psychiatry and to explore the nursing intervention effects. Method: Select 794 schizophrenia patients treated in our hospital from January 2012 to December 2013, make an assessment of nursing risks based on aggressive behaviors in Department of Psychiatry, adopt corresponding nursing intervention measures and observe effects. Results: 320 patients have mild degree of attack risks, 282 patients have moderate degree of attack risks and 192 patients have severe degree of attack risks. After nursing intervention measures are taken, aggressive behaviors have not happened. Conclusion: Nursing personnel should comprehensively master the conditions and mental activities of patients, make conditions assessment to the patients with tendency of violence and take corresponding grade of nursing intervention measures to prevent the attack risks of patients during hospitalization.
\end{abstract}

\author{
KEYWORDS \\ Mental disease \\ Aggressive behaviors \\ Risks assessment
}

\section{Introduction}

Aggressive behaviors refer to the frequently-occurring dangerous behaviors of the patients in Department of Psychiatry during hospitalization, which not only bring the threats to the safety of medical personnel and hospitalized patients but also easily cause nurse-patient dispute [1]. The research makes an accurate nursing assessment to the violent behaviors of 794 schizophrenia patients in our hospital in the past two years with the self-designed "Assessment Sheet of Nursing Risks based on the Aggressive Behaviors of the Patients in Department of Psychiatry", and takes relatively perfect nursing intervention measures according to the assessment results, which has achieved good effects with the report as follows:

Copyright ( 2014 Guolian Zhan

doi: $10.18686 /$ jn.v3i1.3

Received: June 18, 2014; Accepted: August 3, 2014; Published online: October 12, 2014

This is an open-access article distributed under the terms of the Creative Commons Attribution Unported License (http://creativecommons.org/ licenses/by-nc/4.0/), which permits unrestricted use, distribution, and reproduction in any medium, provided the original work is properly cited.

${ }^{\star}$ Corresponding author: Mental Nursing Center of Xiaoshan Hospital of Zhejiang, Hangzhou, Zhejiang 311200, China. E-mail: zgl_1973@163. com

\section{Research objects and methods 2.1. Research objects}

The 794 schizophrenia patients treated in our hospital from January 2012 to December 2013 all conform to the relevant diagnosis standards of CCMD-3. They include 438 males and 356 females with age ranging from 16 to 42 and $26.51 \pm 8.32$ on average.

\subsection{Method}

The self-designed "Assessment Sheet of Nursing Risks based on the Aggressive Behaviors of the Patients in Department of Psychiatry" is applied to assess the violent behaviors of the research objects. The content of the Assessment Sheet includes: (1) whether the patient has the history of wounding people or destroying objects; (2) whether the patient has serious symptoms of paranoia disorder; (3) whether the patient has serious symptoms of delusion of reference; (4) whether the patient is controlled by other mental symptoms; (5) whether the patient has the symptoms of imperative auditory hallucination; (6) whether the patient has serious personality disorder; (7) whether the patient has poor compliance and but strong hatred to medical personnel; (8) whether the patient has unhealthy and stable emotions and are easy to be annoyed; (9) whether the patient has impulse behaviors in the recent one week. 


\subsection{Standards for evaluation}

The full mark is 100 points, including 4 points for each of the first five items and 20 points for each of (6) - (9). The degree of nursing risks: $<9$ points: mild; $10-19$ points: moderate; $\geq 20$ points: serious.

\section{Results}

The assessment result of nursing risks based on violent behaviors of patients in the group shows that: 320 patients have mild degree of attack risks including 176 males and 144 females. They do not cooperate with the treatment, have question, complaint, protest and other passive language attacks, do not believe others and even have hatred to others in communication or think their safety is threatened. 282 patients have moderate degree of attack risks including 164 males and 118 females. Among them, 158 patients have active language attack behaviors including the language attack to medical personnel and other patients, refusing hospitalization and treatment, and destroying objects. The remaining 114 patients do not have attack behaviors within one week before hospitalization and during hospitalization. 192 patients have serious degree of attack risks including 98 males and 94 females. Those patients are easy to be furious, refuse treatment and nursing, do not have compliance and have active attack behaviors. After the targeted nursing intervention measures are taken in the whole group, no attack behavior has arisen.

\section{Nursing}

4.1. Comprehensively collecting medical history, mastering the mental symptoms of patients and offering targeted nursing work

Medical history includes the past medical history, mental symptoms, predisposing factors, personality characteristics and the understanding degree of diseases. Nursing personnel should assess the conditions of patients to learn about whether the inducting factors of attack behaviors exist adopt nursing measures according to the specific conditions and emphasize the work before occurrence of attack behaviors. Nursing personnel should pay special attentions to the high-risk group with male gender, low education level, attack history, unstable emotion, irritability, hallucination, delusion, schizophrenia and mania including their language, behaviors, expressions, posture and tones, and then prevent the occurrence of attack behaviors according to those clues. For the patients with unstable emotion and irritability, nursing personnel should talk with them warmly and considerately rather than rudely, should not quarrel with them or solve their violent behaviors with violence in case of inducing or intensifying attack behaviors. For the patients with obvious hallucination and delusion, nursing personnel should avoid directly involving pathological experience but may induce them to participate in entertainment activities to distract them. Meanwhile, the hazardous articles should be collected and kept well. In the research,
536 patients did not know the truth when being hospitalized and they are easier to conflict with nursing personnel, so nurses should be kind and warm when receiving them $[2,3]$.

\subsection{Building a good nurse-patient work and launch- ing health education}

Nursing personnel should build a good nurse-patient relationship, care and respect them from different aspects, meet their requirements if possible and encourage them to ask medical personnel for help when they are excited and angry; building the good nurse-patient relationship can increase the understanding and trust between nurses and patients, make patients have a happy mood, eliminate the hatred of patients and reduce attack behaviors. Nursing personnel should adopt different forms of health education according to the specific situations of patients, teach patients how to release their anger, such as walking, counting and so on and improve patients' understanding of diseases to promote their confidence in defeating diseases, exert subjective initial and improve their self-protection ability of their health [4].

\subsection{Mental nursing}

Nurses should communicate with patients more, timely find their mental states and offer targeted mental nursing. Before different treatments and nursing, nursing personnel should explain to patients to get their cooperation. For the abnormal talk content of patients, nursing personnel should not show their boredom, denying or other opposite emotions. Nurses should be calm, happy and confident at any time to provide patients with mental support and strengthen their confidence in defeating diseases.

\subsection{Grade nursing}

Different grades of nursing intervention measures should be adopted to the patients with different degrees of attack risks according to risks assessment result. (1) The patients with mild attack risks: nursing personnel should watch their conditions and the possible attack behaviors, and isolate them when necessary. (2) The patients with moderate degree of attack risks: nursing personnel should prevent them from becoming impulsive, isolate them, and keep certain of safety distance from them when contacting with them. (3) The patients with serious degree of risks: nursing personnel should prevent them from being impulsive, regard them as a prevention and nursing emphasis, provide them with 24-hour companion, nursing and protective restraint [5]. The attack behavior of the group is closely related to imperative auditory hallucination and delusion of persecution. For the attack behaviors of patients, nursing personnel should assess the relevant factors and adopt corresponding measures. The 152 patients with imperative auditory hallucination should be isolated in case of the emergent behaviors under the control of auditory hallucination. 
Nursing personnel should adjust the rooms of the 186 patients with serious delusion. "People-oriented management" should be implemented: the bad attitude of medical personnel is also one of the factors causing attacks. There is no attack caused by the bad attitudes of medical personnel in this group. Phones are provided in wards to facilitate the communication with the outside and effectively reduce the attack behaviors caused by the isolation.

\subsection{Medical personnel should strengthen their sense of responsibility and improve predictive ability}

(1) When taking meals and drugs, medical personnel should participate with the management of order. The effective administration method should be adapted to the patients who do not cooperate with treatment. For example, if a patient refuses to take drugs orally and can not be persuaded to take drugs, nursing personnel should report it to the doctor timely and adopt muscle or intravenous injection; if a patient refuses to take drugs orally and is reluctant to take drugs after persuasion, nursing personnel should let the patient take drugs alone, and two or more nursing personnel should be present to check whether the patient really takes drugs and prevent the patient from wounding people out of impulse. (2) Nursing personnel should clear the forbidden objects out and regard it as a routine work. Patients should be checked strictly when entering the hospital to prevent them from bringing forbidden objects (such as glass products, rope, lighter, ringpull can, knife and so on) [6]; in daily work, head nurse should organize nursing personnel to conduct safety checking twice a week and make records; when patients' family members pay a visit, the visitors should be required to receive the checking of the carried objects; meanwhile, hospital should print leaflets and distribute them to family members propagandizing the relevant safety and prevention knowledge when visiting patients. (3) Medical personnel should watch the development of sickness conditions at any time, communicate with patients to learn about their mental states with all attention, and actively adopt prevention measures to prevent violent behaviors, which is the key of safe nursing. For example, nursing personnel can let the patients with hallucination and delusion experience participate in entertainment activities to consume their excessive energies, make exercises and have fun with patients according to their hobbies so as to enhance the trust and understanding with patients, and also help patients to eliminate their bad mood. (4) Nursing personnel should suggest the doctor to provide electroconvulsive treatment for the patients who are in severe tendency to suicide, selfwound or violence [7].

\section{Discussion}

The safe nursing in Department of Psychiatry has an important position in all nursing work, so all medical and nursing personnel shall have high sense of responsibility, scientifically and reasonably apply safety knowledge and skills, be on high alert in the whole nursing work to ensure the safety of patients and workers and the normal proceeding of medical and nursing work. Some materials show that $98.47 \%$ of the nurses in Department of psychiatry have been attacked by patients [8]. The research applies the self-designed "Assessment Sheet of Nursing Risks based on the Aggressive Behaviors of the Patients in Department of Psychiatry" to make an assessment to the hospitalized schizophrenia patients, judges accurately the possibility of violent attacks of each patient and offer targeted nursing intervention measures to reduce the harms of attacks of patients furthest, which can not only effectively prevent the attack behaviors of patients during hospitalization but also has stabilization functions on the patients with attack tendency and attack behaviors to minimum the accident rate of medical personnel.

\section{References}

1. Yao X, Li Z, Xu J. The status survey of risks assessment of aggressive behaviors by the nurses in Department of Psychiatry in Class III Grade I Hospitals. Journal of Nursing Administration. 2011;11(2):108-111.

2. Han Y, Zhao S. The nursing risks assessment and intervention of aggressive behaviors in Department of Psychiatry. Chinese Doctors Community (medical specialty). 2013;15(7):317-318.

3. Li J, Li H, Wang $\mathrm{H}$. The risks assessment and nursing intervention of aggressive behaviors of male schizophrenia patients. Journal of Nurse Training. 2007;22(24):2285-2287.

4. Yan S, Cui H, Zhao Y. The causes analysis and nursing prevention of violent behaviors hospitalized psychosis patients. Medical Journal of Qilu. 2009;24(5):457-458.

5. Yao X, Li Z, Hu L. The relevant factors analysis of the aggressive behaviors of hospitalized psychosis patients. Chinese Journal of Nursing. 2011;46(1):8-11.

6. Liu M, Qiu T. Opinions about the self-prevention of medical personnel from the violent behaviors of psychosis patients. Medical Journal of Chinese People's Health. 2007;19(9):809-810.

7. Jiang $B$. The causes analysis and nursing strategies of violent behaviors of 202 hospitalized psychosis patients. Qinghai Medical Journal. 2012;42(9):39-40.

8. Li F, Gao C. The clinical features and nursing of aggressive behaviors of hospitalized psychosis patients. Chinese Journal of practical nursing. 2003;19(19):47-48. 